\title{
ABASTECIMIENTO DE SEMILLAS DE Nothofagus alessandrii CON CONSIDERACIONES GENÉTICAS
}

\author{
Santelices, Rómulo ${ }^{3}$ Espinoza, Sergio ${ }^{1}$ y Cabrera, Antonio ${ }^{4}$
}

\section{RESUMEN}

Se presenta información y antecedentes de la especie ruil (Nothofagus alessandrii) con el objetivo de orientar e incluir consideraciones genéticas en el abastecimiento de semillas para sus programas de restauración.

Palabras clave: Nothofagus alessandrii, semillas, restauración, consideraciones genéticas.

\section{SUMMARY}

Information and background about ruil (Nothofagus alessandrii) is presented with the aim of to guide and include genetic considerations in the supply of seeds for their restoration programs.

Keywords: Nothofagus alessandrii; seeds; restoration, genetics considerations

\footnotetext{
${ }^{3}$ Universidad Católica del Maule, Facultad de Ciencias Agrarias y Forestales, Centro del Secano

${ }^{4}$ Universidad Católica del Maule, Vicerrectoría de Investigación y Posgrado, Facultad de Ciencias Agrarias y Forestales, Centro del Secano
} 


\section{INTRODUCCIÓN}

La zona mediterránea de Chile, que se extiende desde la Región de Valparaíso hasta la del Bio Bio, se caracteriza por concentrar la mayor biodiversidad del país (Myers et al., 2000). Allí, también se concentra la mayor densidad poblacional de Chile, con la consiguiente presión sobre los recursos naturales.

Como consecuencia, una característica de esta región es la transformación que ha sufrido el paisaje durante los dos últimos siglos, generando como resultado, entre otros aspectos, la reducción y fragmentación de los bosques naturales.

Así, el Bosque Maulino, formación vegetal característica como bosque de transición hacia aquellos más meridionales del tipo templado, ha sido uno de los más afectados y en forma particular lo han sido los bosques de Nothofagus alessandrii Espinosa, especie conocida corrientemente como ruil.

Los bosques de $N$. alessandrii son un ecosistema frágil que a comienzos del siglo XX fueron objeto de tala y quema (Donoso y Landaeta, 1983). Los bosquetes remanentes se encuentran muy fragmentados y están insertos en una matriz de plantaciones con especies alóctonas (Bustamante y Grez, 1995; Bustamante y Castor, 1998).

Considerando la situación de esta especie, hoy tiene una alta prioridad de conservación y el Estado de Chile la ha incorporado en los planes RECOGE (restauración, conservación y gestión), aunque no obstante siguen presentes los procesos antropogénicos que la han llevado a su actual estado de degradación.

Actualmente, está clasificada como una especie rara y en peligro de extinción (D.S. 151/2007 del Ministerio Secretaría General de la Presidencia), e internacionalmente la IUCN la define como en peligro de extinción (Barstow et al., 2017). Es una especie endémica de la Cordillera de la Costa de la Región del Maule (Santelices et al., 2012a).

Uno de los factores de mayor riesgo señalado en el plan RECOGE son los incendios forestales, lo que quedó en evidencia en el periodo estival 2017, con lo que se llamó el incendio de Las Máquinas, que abarcó una superficie cercana a las 180.000 ha, entre ellas parte importante de los bosques de ruil.

Si bien es cierto que una característica de este particular ecosistema es su resiliencia, de hecho, después de casi un año de este disturbio se observa una fuerte rebrotación en la base de los fustes; sigue siendo un sistema muy frágil y amenazado.

Una opción para mejorar su actual condición sugiere la restauración de estos bosques y para ello una alternativa es introducir al sistema brinzales que lo rejuvenezcan y le den mayor vigor. Sin embargo, no existe plena claridad sobre las eventuales poblaciones o subpoblaciones, por lo cual se podría correr un riesgo de contaminación genética al introducir material inadecuado.

No solo la restauración es una de las tareas pendientes, también lo es la regeneración de estos bosques. No obstante, la información disponible para ello es insuficiente y aunque la legislación vigente contempla cierta normativa para estos efectos, ella carece de una base científica que la sostenga.

Por ello, el objetivo de este trabajo es entregar información sobre la especie, que permita orientar el abastecimiento de semillas para programas de restauración, con los debidos resguardos y consideraciones genéticas. 


\section{DESCRIPCIÓN Y DISTRIBUCIÓN DE LA ESPECIE}

El ruil es un árbol caducifolio que puede alcanzar $30 \mathrm{~m}$ de altura y $1 \mathrm{~m}$ de diámetro (Rodríguez et al., 1983; Rodríguez y Quezada, 2003), aunque en la actualidad la mayoría de los ejemplares no supera los $30 \mathrm{~cm}$ de diámetro (Figura $\mathrm{N}^{\circ} 1$ ). La especie tiene una corteza agrietada, con unas manchas blancas muy características, que son originadas por líquenes.

En los individuos jóvenes, con diámetros medios inferiores a $12,5 \mathrm{~cm}$, corresponde a poblaciones de microlíquenes crustáceos Lecanora albella (Pers.) Ach. y en el caso de los adultos y diámetros superiores a $21,5 \mathrm{~cm}$, de cortezas agrietadas y en sitios húmedos, se desarrolla el macrolíquen folioso Parmotrema chinense (Osbeck) Hale \& Ahti. (Pereira y San Martín, 1998).

La copa es frondosa, piramidal y de color verde amarillento, que contrasta con la verde blanquecina de Nothofagus glauca (Phil.) Krasser, especie con la que comparte el hábitat (San Martín et al., 2013). Las hojas de $N$. alessandrii son de lámina simple, de posición alterna a subopuesta en las ramas, con una longitud entre 7 y $13 \mathrm{~cm}$ y un ancho de 4 a $9 \mathrm{~cm}$ (Rodríguez et al., 1983; Rodríguez y Quezada, 2003). La forma es principalmente ovada con margen dentado espinuloso con 11 a 13 notables nervios secundarios rematando cada uno en un diente lateral del borde. El pecíolo es relativamente variable con una longitud que puede variar de 0,5 a $12 \mathrm{~cm}$, e inicialmente es acompañado por estípulas de forma oblonga a lanceoladas, pero que tempranamente se desprenden (San Martín et al., 2013).

Se ha observado que a medida que aumenta la sombra, sus hojas tienden a tener mayor superficie, aunque este efecto no es tan claro en el área foliar específica (Santelices et al., 2012b), lo que podría indicar algún grado de plasticidad para adaptarse a diferentes condiciones de luz en etapas tempranas.

La sexualidad de los individuos es diclina monoica, es decir, tienen flores unisexuales masculinas y femeninas (Rodríguez et al., 1983; Rodríguez y Quezada, 2003). Siguiendo a San Martín et al. (2013), las flores masculinas y femeninas son axilares y se encuentran espacialmente separadas en los extremos superiores de ramas y del borde de la copa.

Las flores masculinas se encuentran en 3 a 4 grupos, portando cada uno 10 a 20 estambres exertos o visibles a simple vista. Las flores femeninas se disponen en inflorescencias sésiles en una cúpula axilar y solitaria.

Cada cúpula porta 3 a 7 flores. En la madurez esta cúpula es piramidal, tetrágona con cuatro valvas aovado - lanceoladas. Cada valva es recubierta por 5-7 apéndices lamelares cortos y en disposición imbricada. Este alto número de flores femeninas indicaría la antigüedad y primitivismo del taxón, respecto a las otras especies del género (Romero, 1986).

Su fruto corresponde a una nuez que contiene entre 3 y 7 semillas dimorfas, cortamente aladas, cónicas y de tres lados las dos laterales y comprimidas las del centro y las valvares (Rodríguez y Quezada, 2003; San Martín et al., 2013).

El tamaño de las semillas es, en general, homogéneo y sólo se han observado diferencias significativas en la longitud de las dímeras y en el ancho de las trímeras. El peso de 1.000 semillas y la capacidad germinativa puede variar significativamente entre las procedencias (Cuadro $\mathrm{N}^{\circ}$ 1) (Santelices et al., 2009) y, en consecuencia, el número de semillas por kilogramo podría hacerlo de las 96.900 a las 140.845 , un rango más amplio al señalado por Donoso y Cabello (1978) de 123.000 a 133.000 semillas por kilogramo. 


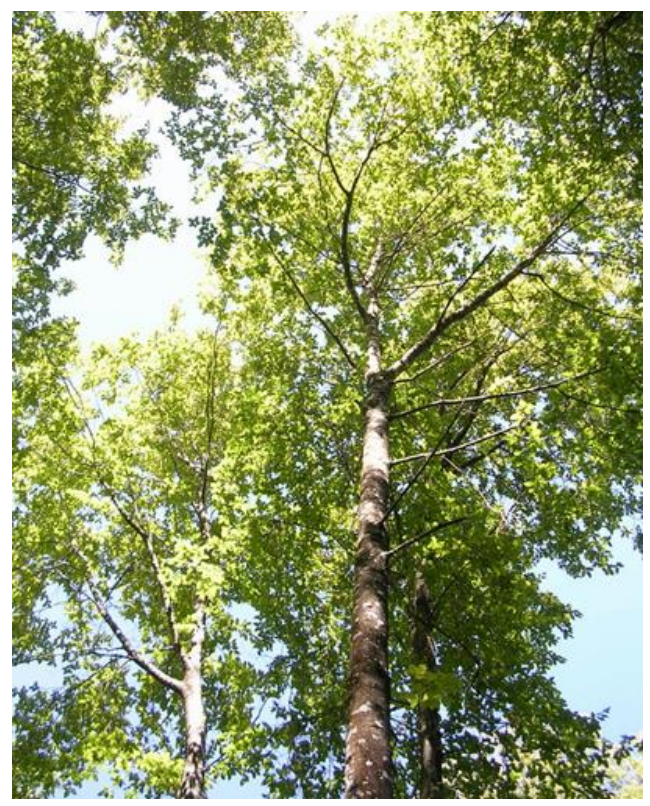

Figura $\mathrm{N}^{\circ} 1$

EJEMPLAR ADULTO DE Nothofagus alessandrii

\section{Cuadro $\mathrm{N}^{\circ} 1$ \\ CARACTERÍSTICAS MORFO-FISIOLÓGICAS DE SEMILLAS DE Nothofagus alessandrii DE DIFERENTES PROCEDENCIAS}

\begin{tabular}{|c|c|c|c|c|c|c|c|}
\hline \multirow{3}{*}{ Procedencia } & \multicolumn{5}{|c|}{$\begin{array}{c}\text { Tamaño de las Semillas } \\
(\mathrm{mm})\end{array}$} & \multirow{3}{*}{$\begin{array}{l}\text { Peso de } \\
1000 \\
\text { Semillas } \\
(g)\end{array}$} & \multirow{3}{*}{$\begin{array}{c}\text { Germinación } \\
(\%)\end{array}$} \\
\hline & \multicolumn{3}{|c|}{ Dímeras } & \multicolumn{2}{|c|}{ Trímeras } & & \\
\hline & Largo & Ancho & Espesor & Largo & Ancho & & \\
\hline Lo Ramírez & $6,2 a b$ & $5,2 \mathrm{a}$ & $1,2 \mathrm{a}$ & $5,8 \mathrm{a}$ & $4,4 \mathrm{~b}$ & $9,43 a b$ & $98,3 \quad a$ \\
\hline Coipué & $6,3 a b$ & $5,3 \mathrm{a}$ & $1,3 \mathrm{a}$ & $6,0 \mathrm{a}$ & $5,0 a b$ & $10,04 a b$ & $78,6 \mathrm{ab}$ \\
\hline Quivolgo & $5,5 \mathrm{~b}$ & $5,1 \mathrm{a}$ & $1,1 \mathrm{a}$ & $5,6 a$ & $4,3 \mathrm{~b}$ & $7,21 \quad \mathrm{c}$ & $76,4 a b$ \\
\hline El Desprecio & $6,1 a b$ & $5,3 \mathrm{a}$ & $1,2 \mathrm{a}$ & $5,8 \mathrm{a}$ & $4,5 \mathrm{~b}$ & $9,11 \mathrm{ab}$ & $96,2 \quad a$ \\
\hline El Corte & $6,4 \quad a$ & $5,4 \mathrm{a}$ & $1,4 \mathrm{a}$ & $6,1 \mathrm{a}$ & $5,7 \mathrm{a}$ & $10,32 \mathrm{a}$ & $45,7 \quad b$ \\
\hline
\end{tabular}

(Fuente: Santelices et al., 2009). Letras distintas misma columna indican diferencias significativas $p<0,05$.

Los bosquetes remanentes de $N$. alessandrii se distribuyen naturalmente sólo en la Cordillera de la Costa de la Región del Maule, en dos grandes zonas interfluviales (Figura $N^{\circ} 2$ ) (Santelices et al., 2012a). La primera de ellas entre los ríos Mataquito y Maule y la segunda entre el Maule y el Curanilahue. La mayor humedad, la influencia marítima, el volumen de las precipitaciones, la orientación y la radiación solar indirecta y su menor efecto de insolación refleja que, en la actualidad, los bosques de ruil responden a otras condiciones de mayor humedad de carácter microclimático azonal como fue en el pasado y no al macroclima regional de carácter zonal. En efecto, su distribución es discontinua y relegada a sólo algunas laderas de exposición de umbría, en sitios de refugio en los que las quebradas representan un corredor para las neblinas vespertinas y matinales, así como del frío y húmedo viento marino (San Martín et al., 2013), 
favoreciendo los requerimientos de humedad de la especie. Si bien es cierto que se ha reportado una superficie remanente de 314 ha, los incendios del periodo estival del año 2017 afectaron parte importante de este recurso, estimándose cerca de un 55\% afectado por el fuego (Valencia et al., 2018).

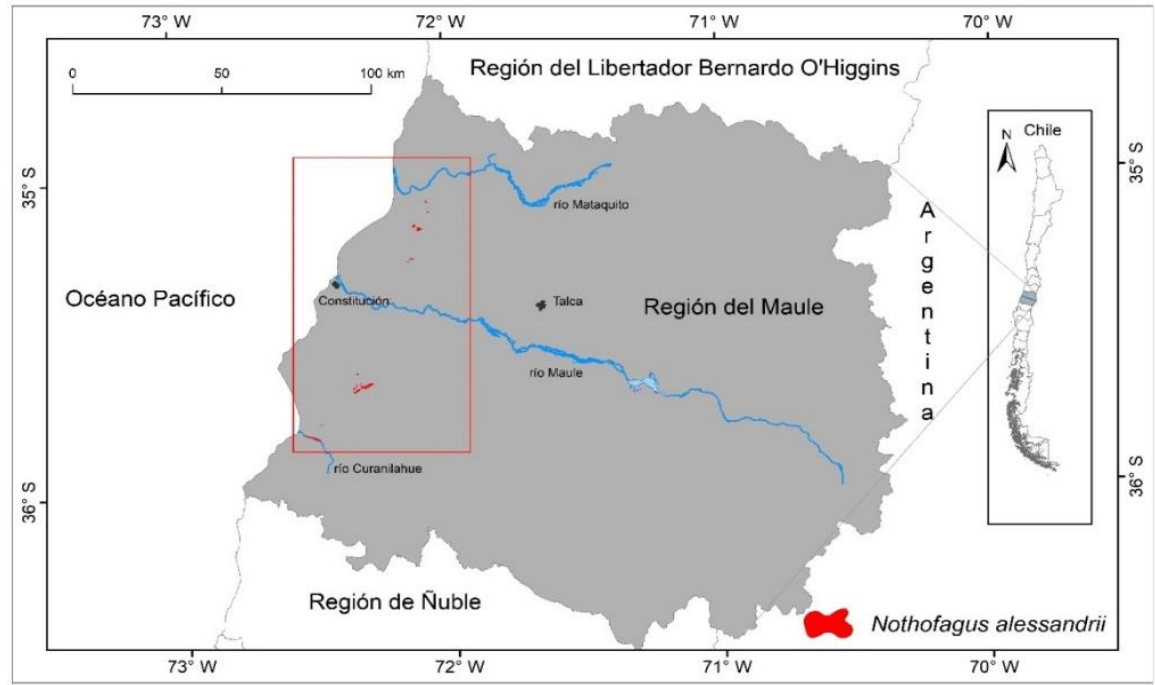

(Fuente: Modificado de Santelices et al., 2012a)

Figura $\mathrm{N}^{\circ} 2$

DISTRIBUCIÓN ESPACIAL DE LOS BOSQUETES DE Nothofagus alessandrii AUTOECOLOGÍA DE LA REGENERACIÓN

De acuerdo a lo señalado por San Martín et al. (2013) y Rodríguez et al. (1983), las flores de $N$. alessandrii son inconspicuas, sexualmente diclinas y axilarmente ubicadas en los extremos terminales de las ramas de un mismo individuo. El carácter primitivo de sus estructuras, prácticamente carentes de envolturas protectoras, significa que no sean vistosas y la polinización sea fundamentalmente por el viento.

La hinchazón de las yemas florales se inicia a mediados de agosto y su explosión a fines del mismo mes e inicio de septiembre dando paso a la antesis o floración. La floración ocurre poco antes de la formación de las hojas y se extiende desde fines de agosto a principios de octubre, con mayor expresión a mitad de septiembre. Este hecho es coincidente con el estado de reposo de $N$. glauca, el cual expande sus yemas e inicia la floración cuando ya $N$. alessandrii ha desarrollado completamente su follaje. Tal asincronía evita una sobreposición de una lluvia polínica (San Martín et al., 2013).

La fructificación y madurez de las semillas se inicia a fines de enero e inicios de febrero del año siguiente (Figura $\mathrm{N}^{\circ} 3$ ). 


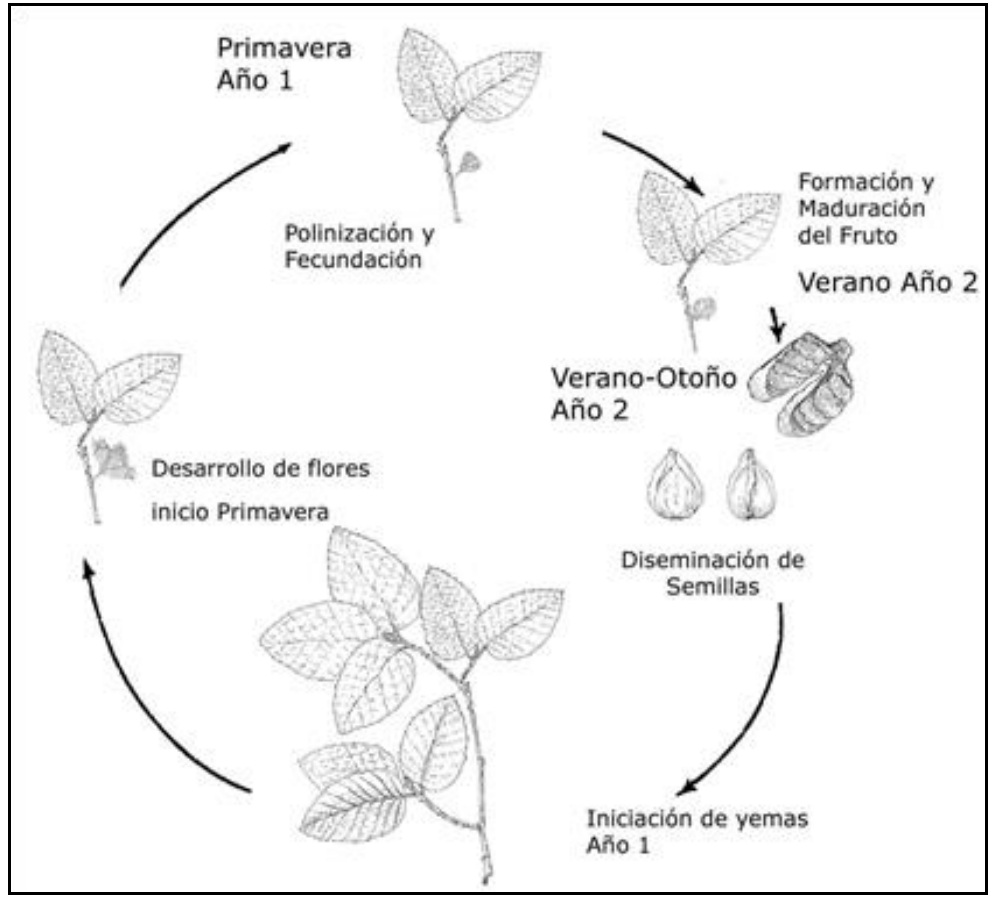

(Fuente: San Martín et al., 2013)

Figura $N^{\circ} 3$

CICLO BIANUAL DE REPRODUCCIÓN DE Nothofagus alessandrii

La morfología de las semillas (Figura $N^{\circ} 4$ ) determina que el patrón de dispersión sea muy pobre y quede fundamentalmente reducido al medio terrestre aéreo-seco y no acuático o marino. Ello condiciona que este sea, principalmente, por gravedad (Hill y Jordan, 1993) y en una pequeña proporción por el viento.

Debido al tamaño de las alas y al obstáculo de árboles vecinos de la matriz, la anemocoría no es eficiente (San Martín et al., 2013).

La diseminación de las semillas se inicia a fines de enero y se prolonga hasta febrero, incluso marzo en su distribución más meridional. La mayor caída ocurre en días iluminados con alta temperatura y con suave brisa. Esta lluvia de nueces con sus semillas es posible advertirla en el interior del bosque.

La producción de semillas se concentra en aquellas partes de la copa de los árboles expuestas a una mayor luminosidad, encontrándose también árboles con nula producción. 


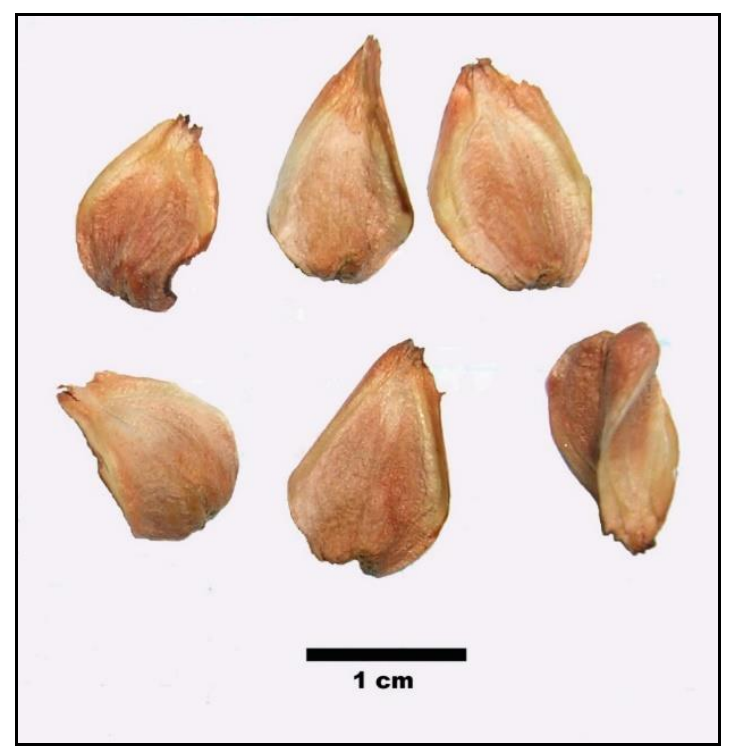

Figura $\mathrm{N}^{\circ} 4$

MORFOLOGÍA DE LAS SEMILLAS DE Nothofagus alessandrii

La colección de semillas es posible hacerla en forma manual y directamente del piso. Una forma práctica de cosecha consiste en extender mallas de plástico antes de la diseminación, lo que ayuda a recogerlas. Posterior a la limpieza y selección de las semillas es necesario asegurar su almacenamiento, para ello es recomendable utilizar envases de vidrio y mantenerlos en refrigeración a $4^{\circ} \mathrm{C}$. Se desconoce el efecto del almacenamiento de las semillas en cuanto a la pérdida natural de viabilidad en el tiempo.

En la regeneración natural por monte alto, la germinación ocurre en los sitios de los rodales potenciados por la exposición del húmedo y frío invierno (San Martín et al., 2013). Se ha establecido que las semillas de $N$. alessandrii tienen una latencia endógena (Santelices et al., 2011b), la que fácilmente se supera con tratamientos pregerminativos, fundamentalmente mediante las técnicas de estratificación fría durante 30 o 45 días o remojo en ácido giberélico en concentraciones de 100 o $200 \mathrm{mg} / \mathrm{L}$ (Santelices et al., 2011b).

Si bien es cierto que fácilmente se rompe la latencia de las semillas viables, llegando a valores que pueden superar un $84 \%$ de capacidad germinativa con la estratificación durante 30 días (Santelices et al., 2011b), la mayor dificultad se relaciona con la alta proporción de semillas infértiles, llegando en algunos casos a superar el $93 \%$, siendo las valvares todas vanas (Santelices et al., 2011a).

No obstante, considerando los diferentes reportes sobre esta materia (Donoso, 1975; Donoso y Cabello, 1978; San Martín et al., 2013), y que es una especie vecera, estos valores podrían variar en función del año de cosecha y de la procedencia de las semillas.

Ruil es una especie que presenta un patrón de germinación epigea, es decir, los cotiledones emergen del suelo como consecuencia del crecimiento del hipocotilo. Si bien es cierto que en su medio natural se ha observado en algunos casos un buen potencial germinativo de la cama de semillas, el repoblado no llega a establecerse debido a que las tasas de reclutamiento son 
muy bajas. En ello influyen, principalmente, factores físicos de tipo mecánico y ecofisiológicos del sitio (San Martín et al., 2013).

En el establecimiento de plantaciones se ha observado que el factor limitante es la protección de los brinzales de la radiación solar directa, para lo cual se han empleado exitosamente mallas de plástico (Quiroz et al., 2009; Santelices et al., 2012b). No obstante, como consecuencia del cambio climático y por ello el consecuente aumento de las temperaturas estivales y de periodos de sequía más prolongados, se ha favorecido el estrés hídrico y una sequía edáfica, lo que redunda en una mayor dificultad en el establecimiento de las plantas en campo.

En relación con lo antes expuesto, a modo de ejemplo se pueden señalar dos casos en los cuales el establecimiento de $N$. alessandrii bajo diferentes condiciones y objetivos fue nulo (datos no publicados).

En la Reserva Nacional Los Ruiles, en el sector El Corte, en la distribución más austral de la especie, en el año 2011 se enriqueció bajo diferentes condiciones de luminosidad con plantas del tipo $1 / 0$ a fines del invierno. A la entrada del otoño del año siguiente en forma visual se monitoreó la plantación, constatando que había una supervivencia de un $100 \%$ y que los brinzales habían doblado su altura inicial. No obstante, en la siguiente primavera se registró una mortalidad total de las plantas. Es probable que este hecho se deba al temperamento que tiene la especie, que tiende a desarrollarse en ambientes húmedos, y que la humedad edáfica posterior al verano no haya sido suficiente para la supervivencia de las plantas, antes de la llegada de las lluvias en invierno.

En otro ensayo, realizado en su área de distribución más septentrional, se estableció una plantación a fines del invierno de 2016, considerando como tratamientos el uso de tubos protectores y plantas a plena luminosidad. A mediados del verano del año siguiente, también se registró una mortalidad del $100 \%$ de las plantas establecidas a plena luminosidad, sin que ellas pudieran siquiera desarrollar un crecimiento en biomasa radical y aérea. Las altas temperaturas registradas, más la prolongada sequía observada en esta zona, probablemente incidieron en que las plantas estuvieran sometidas a un grado de estrés severo, impidiéndoles crecer y superar esta condición.

Si bien es cierto que la regeneración por monte alto no está exenta de dificultades, la estrategia reproductiva que, hasta hoy, ha conducido a la sobrevivencia y resistencia de $N$. alessandrii a una extinción, es de tipo vegetativa (San Martín et al., 2013). Es frecuente la existencia de bosques de segundo crecimiento, probablemente en toda la población de la especie, originados de rebrotes de los tocones. Se ha observado la existencia de numerosos vástagos correspondientes a un solo individuo, abarcando un diámetro superior a los $2 \mathrm{~m}$, y por ello se podría hipotetizar que la especie tiene la capacidad de autoclonación, regenerándose a partir de las raíces.

\section{VARIACIÓN CLINAL O ECOTÍPICA}

Conocer la variabilidad de una especie debería ser el paso previo a la cosecha de semillas para la producción de plantas destinadas a la reforestación y para la generación de eventuales programas de mejoramiento genético (Donoso, 1979). Una posible fuente de variación se debe a la plasticidad del fenotipo frente a un medioambiente variado, pudiendo ello ser una consecuencia de la adaptación genética de poblaciones de una especie a ambientes diferentes, lo que se conoce como genecología, lo que a su vez plantea los conceptos de ecotipo o raza ecológica y de ecoclina.

Si la variación genotípica es continua es tendrá una ecoclina y si es discontinua ecotipos 
o razas ecológicas (Donoso, 1987). Es esperable que a través de una gradiente como la latitud o la altitud se produzcan variaciones, ya sea del tipo clinal o ecotípica.

En Chile, hay especies que tienen una amplia distribución geográfica y en diferentes condiciones climáticas, en donde destacan las del género Nothofagus. Así, por ejemplo, se ha podido determinar que las semillas y flores de Nothofagus obliqua (Mirb.) Oerst. tienen una variación clinal en función de su distribución latitudinal y altitudinal (Donoso, 1987).

La distribución natural de $N$. alessandrii es geográficamente restringida y discontinua. Se le encuentra sólo en sitios específicos en laderas con exposición de umbría en la Cordillera de la Costa de la Región del Maule de Chile. Las condiciones ambientales son relativamente homogéneas, existiendo poca variación en la precipitación y en la temperatura media anual (Santibáñez y Uribe, 1993). Las 314 ha reportadas por Santelices et al. (2012a) se distribuyen desde los $35^{\circ} 05^{\prime} \mathrm{S}$ a los $35^{\circ} 50^{\prime} \mathrm{S}$, es decir, en una franja de no más de $100 \mathrm{~km}$ de extensión latitudinal y en altitudes que van desde los $100 \mathrm{msnm}$ a los $412 \mathrm{msnm}$.

En este contexto, de un paisaje relativamente homogéneo desde el punto de vista geográfico y climático, lo esperable sería no encontrar variaciones, ya sean ecotípicas o clinales, en la distribución de ruil. Sin embargo, en un estudio sobre la producción de semillas de Nothofagus nervosa (Phil.) Dim. et Mil. en un área de distribución de esta especie en Argentina, que es pequeña y comparable a la de ruil, se observaron diferencias importantes en el peso de las semillas en función de su origen y de los años de colecta. En aquellos años de mayor producción las semillas fueron más grandes y pesadas, observándose una variación latitudinal del tipo clinal, con semillas más pesadas en la zona norte de distribución (Marchelli y Gallo, 1999).

A pesar de distribuirse en un hábitat relativamente homogéneo, se ha observado que la procedencia más meridional de $N$. alessandrii podría corresponder a un ecotipo diferente (Santelices et al., 2009) y que las semillas de árboles ubicados en el fondo de quebradas, son más grandes y pesadas que las de aquellos en posiciones más altas e iluminadas (San Martín et al., 2013), probablemente debido a condiciones ambientales diferentes que favorecen la fructificación y semillación.

En un estudio que tuvo como objetivo analizar y caracterizar algunos parámetros cualitativos y cuantitativos de las semillas de 5 procedencias representativas del área de distribución natural de $N$. alessandrii y su comportamiento en vivero, se mostró evidencia de que la procedencia más austral tiende a diferenciarse de las otras $y$, probablemente, corresponda a un ecotipo diferente (Santelices et al., 2009).

Para ello, estos autores determinaron el tamaño, forma, peso y capacidad germinativa de las semillas de las 5 procedencias analizadas. Además, cultivaron plantas en vivero y evaluaron su desempeño mediante los atributos diámetro y altura. Así, Santelices et al. (2009) observaron que el tamaño de las semillas es en general homogéneo y que solo se observan diferencias estadísticas en la longitud de las dímeras y en el ancho de las trímeras; que el peso de 1.000 semillas y la capacidad germinativa varió significativamente entre las procedencias; y que no se registraron diferencias en el diámetro de cuello de la raíz ni en la altura que alcanzaron las plantas después de una temporada de cultivo (Cuadro $\mathrm{N}^{\circ} 1$ ).

También observaron los autores mencionados que el desarrollo de la germinación fue dispar entre las procedencias y que la velocidad de germinación no siempre estuvo asociada a la capacidad germinativa. Así, por ejemplo, la pendiente de la curva de germinación de las semillas de la procedencia más septentrional fue una de las más suaves y con este origen geográfico se obtuvo la mayor capacidad germinativa. En cambio, en las semillas de la procedencia más meridional, durante los diez primeros días se observó una alta velocidad de germinación, para luego decrecer y cambiar muy paulatinamente. Por ello, estos autores concluyen que $N$. alessandrii tiene un comportamiento diferente al de otras especies de su género, sin que se observe una 
variación de tipo clinal, aunque la procedencia más austral (El Corte en la Reserva Nacional Los Ruiles), podría corresponder a un ecotipo diferente. Al no existir una evidencia de que existan ecotipos diferentes, en la producción de plantas es necesario respetar el origen geográfico de la semilla y así evitar la contaminación genética.

\section{DIVERSIDAD GENÉTICA Y SUS IMPLICANCIAS EN LA CONSERVACIÓN DE LA ESPECIE}

La diversidad genética es el componente más básico de la biodiversidad y corresponde a las variaciones heredables que ocurren en cada organismo, entre los individuos de una población y entre las poblaciones dentro de una especie. Como componente esencial de la biodiversidad debe contemplarse su conservación y mantenimiento, en especial en aquellas especies amenazadas, como es el caso de $N$. alessandrii.

Este conocimiento permite, entre otros aspectos, evaluar la capacidad de respuesta de las poblaciones y especies ante los cambios ambientales y desarrollar estrategias de aprovechamiento y conservación de poblaciones, especies y recursos genéticos. Además, el conocimiento de la diversidad genética permite orientar las técnicas de restauración y recuperación de bosques degradados, y en general en las iniciativas de forestación o reforestación con especies nativas.

No obstante, y a pesar de que $N$. alessandrii es una especie con serios problemas de conservación y altamente degradada y fragmentada, la documentación reportada sobre estudios genéticos es aún insuficiente. A lo anterior se suma la ocurrencia del gran incendio del año 2017, que en la Región del Maule consumió parte importante de los bosquetes remanentes de la especie (Valencia et al., 2018).

Debido a lo anterior y desde el punto de vista de la diversidad genética y conservación de recursos genéticos de la especie, surgen algunas interrogantes como ¿Cuáles son los niveles de diversidad genética de las poblaciones de la especie? ¿Qué tipo de manejo debería implementarse para mantener y/o aumentar dicha diversidad? ¿Cuáles poblaciones debieran ser priorizadas para su conservación? ¿Aquellas poblaciones que están ubicadas en las cercanías de centros poblados y centros de consumo, aquellas más pequeñas con presencia de alelos raros, o aquellas más grandes con mayor diversidad?

Estas interrogantes podrían ser respondidas si se cuenta con una adecuada descripción de la diversidad y estructura genética de las actuales poblaciones de ruil.

Es bien sabido que aquellas especies que poseen rangos de distribución estrechos, tal como N. alessandrii, poseen bajos niveles de diversidad genética (Hamrick et al., 1979), debido a la fragmentación del hábitat. Sin embargo, se ha demostrado que algunas especies endémicas, con un rango de distribución restringido, pueden llegar a tener niveles de diversidad moderados a altos (González-Astorga y Castillo-Campos, 2004; Premoli et al., 2001).

En especies del género Nothofagus se han efectuado diversos trabajos tendientes a determinar los niveles de diversidad genética con diferentes marcadores moleculares como isoenzimas o marcadores de ADN (Acosta y Premoli, 2010; Gallo et al., 2006; Marchelli y Gallo, 2001; Mathiasen y Premoli, 2010; Mattioni et al., 2002; Premoli, 1997; Premoli et al., 2012; Vergara et al., 2014). Sin embargo, para $N$. alessandrii sólo se tienen las referencias de Pineda (1998), Torres-Díaz et al. (2007) y Martín et al. (2010), cuyos resultados se resumen en el Cuadro № 2 y se comparan con los resultados obtenidos en especies con rangos de distribución restringido como $N$. alessandrii. 


\section{Cuadro $\mathrm{N}^{\circ} 2$}

INDICADORES DE DIVERSIDAD Y DIFERENCIACIÓN GENÉTICA DE $N$. alessandrii (ANTES DEL INCENDIO DE 2017) Y OTRAS ESPECIES DEL MISMO GÉNERO CON DISTRIBUCIÓN RESTRINGIDA

\begin{tabular}{|l|l|c|r|r|l|}
\hline Especie & Marcador Utilizado & \multicolumn{1}{|c|}{$\mathbf{A}$} & \multicolumn{1}{|c|}{$\mathbf{H}_{\mathbf{E}}$} & \multicolumn{1}{|c|}{$\mathbf{F}_{\mathbf{S T}}$} & \multicolumn{1}{|c|}{ Referencia } \\
\hline \multirow{3}{*}{ Nothofagus alessandrii } & Isoenzima & $2-4$ & -- & 0,15 & Pineda (1998) \\
\cline { 2 - 6 } & Alozimas & 1,8 & 0,18 & ${ }^{*} 0,06$ & Torres-Díaz et al. (2007) \\
\cline { 2 - 6 } & Proteínas de reserva & -- & 0,12 & -- & Martín et al. (2010) \\
\hline Nothofagus nitida & Alozimas & 1,3 & 0,04 & 0,04 & Premoli (1997) \\
\hline Nothofagus moorei & Microsatelites & 1,8 & 0,16 & 0,10 & Taylor et al. (2005) \\
\hline
\end{tabular}

$\mathrm{A}=$ número de alelos por locus; $\mathrm{H}_{\mathrm{E}}=$ heterocigocidad esperada; $\mathrm{F}_{\mathrm{ST}}=$ diferenciación genética entre poblaciones. ${ }^{*}=$ Estimado mediante parámetro $D_{\mathrm{ST}}$.

Desde el punto de la diversidad genética se puede señalar que la de $N$. alessandrii es alta (parámetro $\mathrm{H}_{\mathrm{E}}$ ); aunque se detecta una escasez de heterocigotos y ciertos niveles de endogamia (Pineda, 1998; Torres-Díaz et al., 2007); y que el número de alelos por locus es similar a lo reportado para otras especies del género Nothofagus. Por otra parte, la diferenciación genética entre poblaciones es baja (estimada mediante el parámetro $D_{\mathrm{ST}}$ ) (Torres-Díaz et al., 2007), indicando que los fragmentos de la especie no están diferenciados y que del total de diferencias genéticas entre las poblaciones analizadas, sólo el $6 \%$ es imputable a diferencias entre poblaciones.

No obstante, Pineda (1998) sugiere que existen al menos dos poblaciones genéticamente diferentes, lo que corrobora la necesidad de extender y consensuar los estudios en esta materia. Sin embargo, dada la falta de conectividad entre los fragmentos y la baja de heterocigotos, es esperable que las poblaciones sí estén diferenciadas.

Si bien los resultados anteriores podrían entregar una contribución preliminar a los programas de conservación de $N$. alessandrii, estos deben ser interpretados con cautela debido a que en ambos estudios con la especie se utilizó una baja densidad de marcadores moleculares y un bajo número de loci e individuos. Por ello se debería profundizar en el uso de técnicas más modernas (i.e., microsatelites, SNPs, entre otros) y considerar además abarcar una mayor representatividad del rango de distribución de la especie.

Teniendo un mayor conocimiento de la diversidad y estructura genética de las diferentes poblaciones de $N$. alessandrii se podrían diseñar programas de conservación y restauración específicos para la especie. Para que una población sea exitosa en el largo plazo es necesario que los árboles del lugar presenten suficiente diversidad genética para hacer frente a las condiciones del cambio climático.

En el caso de ruil, y antes de la ocurrencia de los incendios del año 2017, los reportes sobre esta materia indican que esto parece ser posible; sin embargo, los reales efectos de los incendios sobre la diversidad de la especie son aún desconocidos. Por otra parte, y a la hora de restaurar o recuperar un área, y dado que los individuos de un sitio en particular presentan adaptación a las condiciones locales donde habitan (especialmente en especies con un rango de distribución restringido como $N$. alessandrii), es necesario repoblar con organismos procedentes de la misma localidad cuando esto sea posible; no obstante, en situaciones extremas en las cuales se ha perdido gran parte o la totalidad de los individuos de una población, se debe ser más flexible y pensar en la alternativa de trasferencia de material de otras zonas (Broadhurst y Boshier, 2014).

Si lo anterior es posible, sería interesante implementar experimentos de trasplante recíproco para evaluar el fitness de poblaciones locales e introducidas en los distintos sitios de 
origen geográfico, junto con ensayos que imiten la regeneración natural mediante el establecimiento de plantas a altas densidades para promover la competencia.

Actualmente se desconoce si, producto de los incendios, el número de individuos reproductivos que contribuye con descendencia a la siguiente generación (parámetro $\mathrm{Ne}$ ) disminuyó fuertemente o se mantuvo igual. Santelices et al. (2018) plantean una serie de actividades orientadas a reducir la pérdida de diversidad genética y contribuir a aumentar el $\mathrm{Ne}$. Dentro de estas destaca la posibilidad de estudiar el flujo génico y la transferencia de polen entre los distintos fragmentos. $N$. alessandrii es una especie con polinización anemófila y el estudio de Torres-Díaz et al. (2007) señala que hay un flujo polínico muy bajo entre fragmentos de la especie (parámetro $\mathrm{N}_{\mathrm{m}}<1$ ).

Dado que la especie está altamente fragmentada es esperable que el nivel de fragmentación disminuya el flujo génico y, si esto es así, los efectos deletéreos a largo plazo pueden traer graves consecuencias para la diversidad de la especie. Aunque se asume que el sistema de compatibilidad reproductiva de la especie es similar a sus parientes más cercanos, este no es bien conocido y esta tarea también resulta fundamental a la hora de definir estrategias de conservación para la especie.

Finalmente, y si bien se necesitan menos de 400 individuos reproductivos para proveer abundante semilla (Broadhurst y Boshier, 2014), en el caso de N. alessandrii, esto puede resultar complejo en el largo plazo. Las diversas campañas de colecta de semilla, junto con el efecto negativo de los incendios (i.e., quema de árboles madre), indican una baja sostenida en la producción de semilla de la especie y justifican la sugerencia de Santelices et al. (2018) de evaluar el efecto de inductores de la floración a base de reguladores de crecimiento.

\section{CONSIDERACIONES PARA EL ABASTECIMIENTO DE SEMILLAS}

A la luz de los estudios genéticos publicados sobre la especie, no es posible todavía asegurar si en su distribución natural se encuentran una o dos poblaciones de $N$. alessandrii. Eventualmente, la distribución más meridional podría ser una población diferente a las de más al norte. Por otra parte, en la producción de plantas en vivero se ha observado una baja viabilidad de las semillas, incluso menor a un 10\%, y también es frecuente observar la generación de plantas enanas, que solo se mantienen vivas gracias a las condiciones favorables propias del cultivo en vivero (en condiciones naturales no debieran sobrevivir). Estos dos hechos podrían ser indicadores de que la especie está sujeta a endogamia.

La depresión endogámica se expresa en una menor producción de semilla, un peso menor en la semilla, o viabilidad reducida del polen. Adicionalmente se observa una disminución en la resistencia a los factores de estrés, como enfermedades y temperaturas extremas. En consecuencia, es necesario profundizar en este tipo de estudios con técnicas más modernas que permitan discriminar las eventuales poblaciones de la especie y su diversidad genética.

Por otra parte, con información limitada sobre la extensión y escala de la variación adaptativa en árboles nativos, la discusión sobre fuentes de semillas adecuadas a menudo enfatiza lo "local" en un sentido muy estrecho o dentro de límites políticos, en lugar de basarse en pruebas sólidas de la escala sobre la cual ocurre la adaptación (Broadhurst y Boshier, 2014). En consecuencia, conociendo la estructura genética de las eventuales diferentes poblaciones de $N$. alessandrii se podrían diseñar programas de conservación específicos.

La introducción de una especie a menudo es producto de una cosecha limitada sobre un cierto número de poblaciones base y se acompaña inevitablemente de una reducción de la diversidad total de la especie en su área natural. Vander Mijnsbrugge et al. (2010) señalan que es necesario conocer la diversidad genética del material a introducir para evitar problemas como mala adaptación a condiciones locales y un bajo fitness, hibridación intraespecífica y la resultante 
depresión por endogamia, y superioridad de los genotipos introducidos por sobre los locales, hasta convertirse en invasores del área.

En este contexto, la tendencia es sugerir que se trabaje solo con semillas locales, pero ¿qué se entiende por semillas locales? Broadhurst y Boshier (2014) plantean las siguientes interrogantes ¿Que sean del mismo bosque, de la misma cuenca hidrográfica, de la misma provincia, o del mismo país? ¿Qué es más importante, la distancia ecológica o la geográfica? Montalvo y Ellstrand (2000) sostienen que el éxito a largo plazo de poblaciones restauradas puede verse expuesto a riesgos debido al origen del material vegetal si este no corresponde adecuadamente a su nuevo ambiente. Por ello, estos autores hipotetizan que las ventajas del sitio predicen que el éxito relativo de poblaciones introducidas decrecerá al incrementarse su distancia genética y ambiental de la población nativa local.

El requisito de utilizar semillas recolectadas localmente ha tenido tal prioridad que los proyectos de restauración se han abandonado ocasionalmente debido a la falta de fuentes de semillas locales apropiadas (Wilkinson, 2001). Considerando que en el verano de 2017 se quemó casi un $55 \%$ de los bosquetes remanentes de ruil, y que solo algunos bosquetes de las comunas de Curepto (más septentrional) y de Chanco (más meridional), no fueron afectados por los incendios, debe priorizarse la conservación de este recurso genético.

Gracias a la resiliencia que tiene este ecosistema, la especie se ha mantenido a lo largo del tiempo por su capacidad para rebrotar y es lo que hoy está sucediendo. No obstante, en un escenario de cambio climático debería evaluarse en el tiempo la viabilidad de esta recuperación y programar la restauración activa del sistema con una fuente apropiada de semillas. Así, por ejemplo, aquellos bosquetes afectados por el fuego ubicados al norte del río Maule, podrían ser restaurados con semillas de las localidades de Agua Buena, Coipué, Lo Ramírez, y Macal. En cambio, en todos aquellos bosquetes localizados en la comuna de Empedrado debería considerarse el uso de semilla de los escasos árboles que no fueron mayormente afectados por el fuego.

Sin embargo, si la producción de semilla es muy precaria, se debería analizar, desde el punto de vista genético, la alternativa de trasferencia de semilla de otras zonas. No obstante, ante la escasez de semillas también debería pensarse en la producción de plantas mediante propagación vegetativa por estacas (Santelices, 2005; Santelices y García, 2003).

En resumen, mientras no se cuente con una sólida información sobre la diversidad genética de la especie, se debería siempre utilizar semilla local.

\section{REFERENCIAS}

Acosta, M. C. y Premoli, A. C., 2010. Evidence of chloroplast capture in South American Nothofagus (subgenus Nothofagus, Nothofagaceae). Molecular Phylogenetics and Evolution 54: 235-242.

Barstow, M.; Echeverría, C.; Baldwin, H. y Rivers, M. C., 2017. Nothofagus alessandrii. The IUCN Red List of Threatened Species 2017: e.T32033A2808995. Consultado March 2018. Disponible en http://www.iucnredlist.org/details/32033/0.

Broadhurst, L. y Boshier, D. H., 2014. Seed provenance for restoration and management: conserving evolutionary potential and utility. En: Bozzano, M., et al. (eds.). Genetic Considerartions in Ecosystem restoration using native tree species. FAO. p. 27-38.

Bustamante, R. y Grez, A., 1995. Consecuencias ecológicas de la fragmentación de los bosques nativos. Ambiente y Desarrollo 11: 58-63. 
Bustamante, R. O. y Castor, C., 1998. The decline of an endangered temperate ecosystem: the Ruil (Nothofagus alessandrii) forest in central Chile. Biodiversity and Conservation 7: 1607-1626.

Donoso, C., 1975. Aspectos de la fenología y germinación de las especies de Nothofagus de la zona mesomórfica. Fac. Cs. Forest. Universidad de Chile. Santiago, Chile. 32 p.

Donoso, C., 1979. Variación y tipos de diferenciación en poblaciones de roble (Nothofagus obliqua (Mirb.) Oerst.) Bosque 3: 1-14.

Donoso, C. 1987. Variacion Natural en Especies de Nothofagus en Chile. Bosque 8: 85-97.

Donoso, C. y Cabello, A., 1978. Antecedentes fenológicos y de germinación de especies leñosas chilenas. Ciencias Forestales 1: 31-40.

Donoso, C. y Landaeta, E., 1983. Ruil (Nothofagus alessandrii), a threatened Chilean tree species. Environmental Conservation 10: 159-162.

Gallo, L. A.; Marchelli, P.; Azpilicueta, M. M. y Crego, P., 2006. El uso de marcadores genéticos en el género Nothofagus con especial referencia a raulí y roble. Bosque 27: 3-15.

González-Astorga, J. y Castillo-Campos, G., 2004. Genetic variability of the narrow endemic species tree Antirhea aromatica (Rubiaceae, Guettardeae) in a tropical forest of México. Annals of Botany 93: 521-528.

Hamrick, J. L.; Linhart, Y .B. y Mitton, J. B., 1979. Relationships between life history characteristics and electrophoretically-detectable genetic variation in plants. Annual Review of Ecology and Systematics 10: 173-200.

Hill, R. y Jordan, G., 1993. The evolutionary history of Nothofagus (Nothofagaceae). Australian Systematic Botany 6: 111-126.

Marchelli, P. y Gallo, L., 1999. Annual and geographic variation in seed traits of Argentinean populations of Southern Beech Nothofagus nervosa (Phil.) Dim. et Mil. Forest Ecology and Management 121: 239-250.

Marchelli, P. y Gallo, L. A., 2001. Genetic diversity and differentiation in a Southern Beech subjected to introgressive hybridization. Heredity 87: 284-293.

Martín, M. A.; Muñoz, S.; Muñoz, F.; Uribe, M.; Molina, J. R.; Herrera, M. A.; Martín, L. M. y Alvarez, J. B., 2010. Primeros resultados en el desarrollo de un marcador genético basado en las proteínas de reserva en dos especies del género Nothofagus. Bosque 31: 252-257.

Mathiasen, P. y Premoli, A. C., 2010. Out in the cold: genetic variation of Nothofagus pumilio (Nothofagaceae) provides evidence for latitudinally distinct evolutionary histories in austral South America. Molecular Ecology 19: 371-385.

Mattioni, C.; Casasoli, M.; Gonzalez, M.; Ipinza, R. y Villani, F., 2002. Comparison of ISSR and RAPD markers to characterize three Chilean Nothofagus species. Theoretical and Applied Genetics 104: 1064-1070.

Montalvo, A. M. y Ellstrand, N. C., 2000. Transplantation of the Subshrub Lotus scoparius: Testing the HomeSite Advantage Hypothesis. Conservation Biology 14: 1034-1045.

Myers, N.; Mittermeier, R. A.; Mittermeier, C. G.; da Fonseca, G. A. B. y Kent, J., 2000. Biodiversity hotspots for conservation priorities. Nature 403: 853-858.

Pereira, I. y San Martín, J., 1998. Flora liquénica corticícola en un bosque caducifolio de Nothofagus alessandrii de Chile Central. Cryptogamie - Bryologie Lichenologie. Paris 19: 59-72.

Pineda, G., 1998. Determinación de los patrones de variabilidad genética en poblaciones de raulí (Nothofagus alpina (Poepp. et Endl.) Oerst.) y ruil (Nothofagus alessandrii Espinosa), por medio de electroforesis horizontal en geles de almidón. Tesis Memoria Ingeniero Forestal. Facultad de Ciencias Agrarias y Forestales, Universidad de Chile. Santiago, Chile. 79 p.

Premoli, A. C., 1997. Genetic variation in a geographically restricted and two widespread species of South American Nothofagus. Journal of Biogeography 24: 883-892. 
Premoli, A. C.; Acosta, M. C.; Mathiasen, P. y Donoso, C., 2012. Genetic variation in Nothofagus (subgenus Nothofagus). Bosque 33: 115-125.

Premoli, A. C.; Souto, C. P.; Allnutt, T. R. y Newton, A. C., 2001. Effects of population disjunction on isozyme variation in the widespread Pilgerodendron uviferum. Heredity 8: 337-343.

Quiroz, I.; Pincheira, M. y Hernández, A., 2009. Restauración ecológica en la zona central de Chile: Técnicas Silvícolas para la Supervivencia y Crecimiento de Ruil, Hualo, y Peumo bajo Condiciones de Estrés Hídrico. Chile Forestal 363: 50-53.

Rodríguez, R.; Matthei, O. y Quezada, M., 1983. Flora arbórea de Chile. Editorial Universidad de Concepción. Concepción, Chile. 408 p.

Rodríguez, R. y Quezada, M., 2003. Fagaceae. En: Marticorena, C. y Rodríguez, R. (eds.). Flora de Chile 2. Universidad de Concepción. Concepción, Chile. p. 64-76.

Romero, E. J., 1986. Fossil Evidence Regarding the Evolution of Nothofagus Blume. Annals of the Missouri Botanical Garden 73: 276-283.

San Martín, J.; Santelices, R. y Henríquez, R., 2013. Nothofagus alessandrii Espinosa, Ruil. Familia: Nothofagaceae. En: Donoso, C. (ed.). Las Especies Arbóreas de los Bosques Templados de Chile y Argentina: Autoecología. Marisa Cuneo Ediciones. Valdivia, Chile. p. 391-401.

Santelices, R., 2005. Efecto del árbol madre sobre la rizogénesis de Nothofagus alessandrii. Bosque (Valdivia) 26: 133-136.

Santelices, R.; Cerrillo, R. M. N.; Drake, F. y Mena, C., 2011a. Effect of cover and fertilization on the early development of Nothofagus alessandrii nursery container seedlings. Bosque 32: 85-88.

Santelices, R.; Drake, F.; Mena, C.; Ordenes, R. y Navarro-Cerrillo, R. M., 2012a. Current and potential distribution areas for Nothofagus alessandrii, an endangered tree species from central Chile. Ciencia $e$ Investigación Agraria 39: 521-531.

Santelices, R.; Drake, F. y Navarro-Cerrillo, R. M., 2012b. Establishment of a Nothofagus alessandrii plantation using different levels of shade and weed control methods in Talca province, central Chile. Southern Forests 74: 71-76.

Santelices, R.; Espinoza, S.; Cabrera, A. y Magni, C., 2018. Gestión de riesgo como herramienta para la conservación de los bosques de Nothofagus alessandrii, una especie en peligro de extinción de Chile central. Interciencia 43: 144-150.

Santelices, R. y García, C., 2003. Efecto del ácido indolbutírico y la ubicación de la estaca en el rebrote de tocón sobre la rizogénesis de Nothofagus alessandrii Espinosa. Bosque (Valdivia) 24: 53-61.

Santelices, R.; Navarro-Cerrillo, R. M. y Drake, F., 2009. Caracterización del material forestal de reproducción de cinco procedencias de Nothofagus alessandrii Espinosa una especie en peligro de extinción. Interciencia 34: 113-119.

Santelices, R.; Navarro-Cerrillo, R. M. y Drake, F., 2011b. Propagation and seedling cultivation of the endemic species Nothofagus alessandrii Espinosa in Central Chile. Restoration Ecology 19: 177-185.

Santibáñez, F. y Uribe, J., 1993. Atlas Agroclimático de Chile, regiones VI, VII, VIII y IX. Ediciones de la Universidad de Chile. Santiago, Chile. 99 p.

Taylor, K. J.; Lowe, A.; J. Hunter, R.; Ridgway, T.; M. Gresshoff, P. y Rossetto, M., 2005. Genetic diversity and regional identity in the Australian remnant Nothofagus moorei. Australian journal of Botany 53: 437-444.

Torres-Díaz, C.; Ruiz, E.; González, F.; Fuentes, G. y Cavieres, L. A., 2007. Genetic diversity in Nothofagus alessandrii (Fagaceae), an endangered endemic tree species of the coastal maulino forest of central Chile. Annals of Botany 100: 75-82.

Valencia, D.; Saavedra, J.; Brull, J. y Santelices, R., 2018. Severidad del daño causado por los incendios forestales en los bosques remanentes de Nothofagus alessandrii Espinosa en la región del Maule de Chile. Gayana botánica 75: 531-534. 
Vander Mijnsbrugge, K.; Bischoff, A. y Smith, B., 2010. A question of origin: Where and how to collect seed for ecological restoration. Basic and Applied Ecology 11: 300-311.

Vergara, R.; Gitzendanner, M. A.; Soltis, D. E. y Soltis, P. S., 2014. Population genetic structure, genetic diversity, and natural history of the South American species of Nothofagus subgenus Lophozonia (Nothofagaceae) inferred from nuclear microsatellite data. Ecology and Evolution 4: 2450-2471.

Wilkinson, D. M., 2001. Is local provenance important in habitat creation? Journal of Applied Ecology 38: 13711373. 